ITP-SB-99-03, CLNS 99/1610

\title{
Collider Signatures from the Brane World
}

\author{
Gary Shiu $^{1 *}$, Robert Shrock ${ }^{1 \dagger}$ and S.-H. Henry Tye ${ }^{2 \ddagger}$ \\ ${ }^{1}$ Institute for Theoretical Physics, State University of New York, Stony Brook, NY 11794 \\ ${ }^{2}$ Newman Laboratory of Nuclear Studies, Cornell University, Ithaca, NY 14853
}

(April 7, 1999)

\begin{abstract}
We discuss some collider signatures of the brane world. In addition to the usual bulk (closed string) fields and brane (open string) fields in the Type I string picture, there are closed string fields, namely, twisted modes, which are not confined on the branes but nonetheless are localized in the extra compactified dimensions. While the coupling of the Standard Model (brane) fields with a bulk mode (such as the graviton) is suppressed by powers of the Planck mass, their coupling to a twisted mode is suppressed only by powers of the string scale $M_{s}$, which can be as low as a few TeV. This means these localized twisted fields can have important observable effects in the $\mathrm{TeV}$ range, including resonances in dijet invariant mass distributions in $\bar{p} p, p p \rightarrow$ jets + $X$. Given the current lower bound on the fundamental higher-dimensional Planck scale, the experimental effects of these twisted fields may turn out to be larger than the effects of virtual and real KK gravity modes. The collider signatures of anomalous $U(1)$ gauge symmetries as well as other phenomenological implications of the brane world are also discussed.
\end{abstract}

\footnotetext{
*E-mail: shiu@insti.physics.sunysb.edu

†E-mail: shrock@insti.physics.sunysb.edu

‡E-mail: tye@mail.lns.cornell.edu
} 


\section{INTRODUCTION}

It was proposed in [1] that the fundamental Planck scale can be around a TeV. In string theory, this implies that the string scale $M_{s}$ is lowered all the way down to TeV scale [2 4 . In this picture, the Standard Model (SM) fields reside inside of $p \leq 9$ spatial dimensional $p$-branes (or the intersection of different sets of branes, as in [4]), while gravity lives in the higher (10 or 11) dimensional bulk of spacetime. For $3<p<9$, this "brane world" scenario appears to be flexible enough so that various properties such as gauge and gravitational coupling unification, dilaton stabilization, and the weakness of the SM gauge couplings can be satisfied within this framework [5]. The weakness of the four dimensional gravitational coupling is due to the presence of at least two large $\left(\gg 1 / M_{s}\right)$ compact directions transverse to the $p$-branes on which the SM fields are localized. Issues such as coupling unification [7], proton stability [6], neutrino masses [8], fermion masses and mixing [9], and astrophysical/cosmological implications [6,10] have also been addressed [11, 12, 16]. It has been argued that the possibility of $\mathrm{TeV}$ scale gravity appears viable. If the fundamental Planck scale were around a $\mathrm{TeV}$, clear signals due to strong gravitational interactions would appear at the LHC, extending existing constraints [13]. Furthermore, light Kaluza-Klein (KK) modes living in the bulk can be produced.

In general, the conventional Planck mass $M_{P} \sim 10^{19} \mathrm{GeV}$ is related to the string scale by $\left(V_{p-3} V_{9-p} M_{s}^{6}\right)=\left(M_{P} / M_{s}\right)^{2}$, where $V_{p-3}$ and $V_{9-p}$ are the compactified volumes inside and transverse to the $p$-branes respectively. Specifically, it is convenient to discuss the brane world picture in Type I string models, where brane fields are open string modes. The scenario given in Ref [4] is of this type. Here the Planck mass is given by

$$
M_{P} \sim M_{s}\left(M_{s} R_{1}\right)\left(M_{s} R_{2}\right)\left(M_{s} R_{3}\right) \sim M_{P l}\left(M_{P l} R_{1}\right)
$$

where $R_{i}$ are the three radii characterizing the 3 compactified tori and $M_{P l}$ is the 6dimensional Planck mass scale [1], in the case with $n=2$ large dimensions. To stabilize the dilaton expectation value (and maybe even to induce SUSY breaking), the string coupling $g_{s}$ is likely to be strong (or, more precisely, $g_{s} \gtrsim 1$, and the theory appears not to have a dual weak coupling description). For $p=5$, a typical squared gauge coupling $\alpha=g^{2} /(4 \pi)$ is given by $\alpha \sim g_{s} /\left(M_{s} R_{i}\right)^{2}$ for $i=2$ or 3 . To obtain the weak SM gauge couplings from a generic strong string coupling requires that $\left(M_{s} R_{2}\right)\left(M_{s} R_{3}\right) \sim 10-100$. For $n=2$, a constraint from supernova cooling requires $M_{P l}>30-50 \mathrm{TeV}$ 6. 15], implying that $M_{s}>3-20 \mathrm{TeV}$. This value for $M_{s}$ is compatible with the SUSY models [12] and the brane world picture [5]. In this case, neither the Tevatron nor LHC energies may be high enough to detect either the strong gravity effect or the gravity KK modes. Are there other signatures at these energies that may be used to test the brane world scenario? The answer is yes, as we show here.

The collider signatures that we discuss here do not arise from strong gravity or bulk KK modes (as in Refs. [13,14]) but come from two generic features of Type I string theory, which should also be generic in the brane world picture:

- (1) In contrast to perturbative heterotic string theory, in some Type I string models (which can be realized as Type II orientifolds and are dual to non-perturbative heterotic strings), there can be more than one anomalous $U(1)$ gauge fields. They must be massive, though some of them can be relatively light and may be produced in high energy colliders as $Z^{\prime}$ vector bosons. 
- (2) There are closed string modes which are not bulk modes, that is, they do not propagate freely in the bulk. Some of these modes are localized away from the branes so their couplings to the brane modes may be exponentially suppressed. However, some of them may sit on the branes. For $p>3$, some of these modes may even be localized inside the branes, i.e., in the dimensions which are compactified. In contrast to the KK modes (from either the branes or the bulk), these localized modes can be produced as a resonance from the scattering of two brane modes which have no momentum in the compactified dimensions, with a significant (i.e., not gravitational strength) coupling. Some of these localized modes are the pseudo-Goldstone bosons eaten by the anomalous $U(1)$ gauge bosons mentioned above. Experimental constraints require the remaining ones to pick up relatively large masses, but one of them may behave like an axion.

For example, in an orbifold string model, these fields are the twisted sector moduli which are stuck at the orbifold fixed points (or fixed lines). Unlike the untwisted sector states which are free to propagate in the bulk, the twisted sector states have no momentum or winding in the compactified dimensions, and hence are localized in the internal space. While the couplings of the brane fields with the bulk fields are suppressed by a factor of $\left(V_{p-3} V_{9-p} M_{s}^{6}\right)^{-1}=\left(M_{s} / M_{P}\right)^{2}$ due to wavefunction normalization in the compactified dimensions (and hence the couplings are of gravitational strength), this suppression factor (or part of it) is absent in the corresponding coupling with these twisted fields. If these fields happen to sit on the branes, the coupling with the brane fields is simply $\kappa \sim g_{s}$ and is therefore independent of $M_{s}, V_{p-3}$ and $V_{9-p}$. Before SUSY breaking, these fields, being moduli, are massless. However, to be compatible with experiment, these states must pick up masses. Assuming that dynamical SUSY breaking takes place on the branes we expect these twisted fields to obtain masses somewhat comparable to the SUSY breaking scale, $M_{S U S Y} \sim O(1) \mathrm{TeV}$.

In the scattering of two brane modes with no momentum in the compactified dimensions, the brane KK modes cannot be singly produced, since this would violate momentum conservation along the branes. In contrast, the momenta orthogonal to the branes need not be conserved since the branes break translation invariance in these orthogonal directions. Therefore, the bulk KK modes can be singly produced by the brane modes, with a coupling in the amplitude $\propto 1 / M_{P}$. After the summation over the large multiplicity $\sim\left(s^{1 / 2} R\right)^{n}$ of bulk KK modes (where $n$ denotes the number of large compactification dimensions transverse to the branes with size $\sim R$, and $s$ denotes the CM mass energy squared), the cross section for processes involving real bulk KK emission is $\propto 1 / M_{P l}^{n+2}$. For processes involving the exchange of virtual bulk KK modes, summing over the exchanges that do not have strong propagator suppression, the amplitude is $\sim\left(1 / M_{P}^{2}\right)\left(s^{1 / 2} R\right)^{n} \propto 1 / M_{P l}^{n+2}$, and the resultant cross sections are $\propto 1 / M_{P l}^{2(n+2)}$. Given that $M_{P l} \gtrsim 30-50 \mathrm{TeV}(n=2)$, these effects may be too small to be observable at Tevatron or even LHC energies. In contrast to the KK modes, the twisted modes which have no momentum or winding in the compactified dimensions can be singly produced by the brane modes with a coupling $\propto 1 / M_{s}$. They can thus be produced as resonances with strengths that are not suppressed by the size of the extra large dimensions. Despite the fact that the couplings of these twisted fields with the Standard Model fields do not appear at the renomalizable level, and the cross sections for processes involving the exchange of these twisted fields are hence suppressed by the factor $1 / M_{s}^{4}$, their experimental signatures may well be larger than those of the gravity KK modes. 
In a typical Type I string model, there are abelian gauge fields (brane modes) with fieldtheoretic triangle anomalies. They become massive generically, with some of the abovementioned twisted RR scalars being the would-be-Goldstone bosons. If the mass of an abelian gauge field happens to be in the LHC energy regime, it may be seen as a resonance in the hadron scattering experiment and in the Drell-Yan process. Since these massive gauge bosons are brane fields, they couple to the quark-antiquark and lepton-antilepton pair with the strength comparable to that of the SM interactions. In contrast to perturbative heterotic string theory, there can be more than one anomalous $U(1)$ gauge fields for Type I strings. Moreover, these $U(1)$ gauge fields can be relatively light and are possible candidates of the $Z^{\prime}$ bosons.

\section{SOME GENERIC FEATURES OF THE BRANE WORLD}

For illustrative purposes, let us first consider compactification of Type I string theory to $4 \mathrm{D}$ on a $\mathbb{Z}_{3}$ orbifold [17]. The $\mathbb{Z}_{3}$ generator $g$ acts on the complex coordinates $z_{1}, z_{2}$, $z_{3}$ of the compactified dimensions $T^{6}=T^{2} \times T^{2} \times T^{2}$ as follows: $g z_{i}=\omega z_{i}$ for $i=1,2,3$ where $\omega=e^{2 i \pi / 3}$. The resulting model has $\mathcal{N}=1$ supersymmetry. The twisted sector states are located at points in the compactified dimensions that are invariant under the orbifold action. Here there are 27 fixed points at which the $\mathbb{Z}_{3}$ twisted sector states are located: $\left(z_{1}, z_{2}, z_{3}\right)=(1 / \sqrt{3}) e^{\pi i / 6}\left(k_{1} R_{1}, k_{2} R_{2}, k_{3} R_{3}\right)$, where $k_{i}=0,1,2$ and $R_{i}$ are the radii of the three $T^{2}$ 's (in each $T^{2}$, the two radii are the same because of the $\mathbb{Z}_{3}$ orbifold symmetry). The twisted sector states at each fixed point form an $\mathcal{N}=1$ neutral chiral supermultiplet. In particular, the scalar component is $\phi_{k}+i a_{k}$ where $\phi_{k}\left(a_{k}\right)$ comes from the NS-NS (R-R) sector.

Consistency (i.e., tadpole cancellation) requires introducing open string and 32 D9branes. In the T-dual picture where two of the complex dimensions $z_{1}$ and $z_{2}$ are dualized, the $D 9$-branes become $D 5$-branes. The gauge group is maximal when the $D 5$-branes are sitting on top on each other, for instance, when they are all located at $z_{1}=z_{2}=0$. In this case, the model has $S O(8) \times S U(12) \times U(1)$ gauge symmetry. The open string sector gives rise to three families of charged chiral multiplets in the $(\mathbf{8}, \overline{\mathbf{1 2}})(-1)$ and $(\mathbf{1}, \mathbf{6 6})(+2)$ representations. In this brane configuration, not all the twisted sector states are located on the branes. In particular, out of the 27 twisted sector chiral multiplets, only 3 of them (the ones with $k_{1}=k_{2}=0$ ) are located on the branes. The remaining twisted sector states are located at a non-zero distance from the branes.

The strength of the coupling of a twisted field with the brane fields depends on the number of dimensions in which the twisted field lives, and its location from the branes. In the above example, the $\mathbb{Z}_{3}$ twisted sector states live in four dimensions and so for the corresponding fixed points which lie on the branes, the couplings with the brane modes are unsuppressed, i.e., $\kappa \sim g_{s}$. For the remaining twisted moduli located at non-zero distances $|X|$ from the branes, the corresponding couplings with the brane fields are exponentially suppressed as $\exp \left(-\left(M_{s}|X|\right)^{2}\right)$. 


\section{FIGURES}

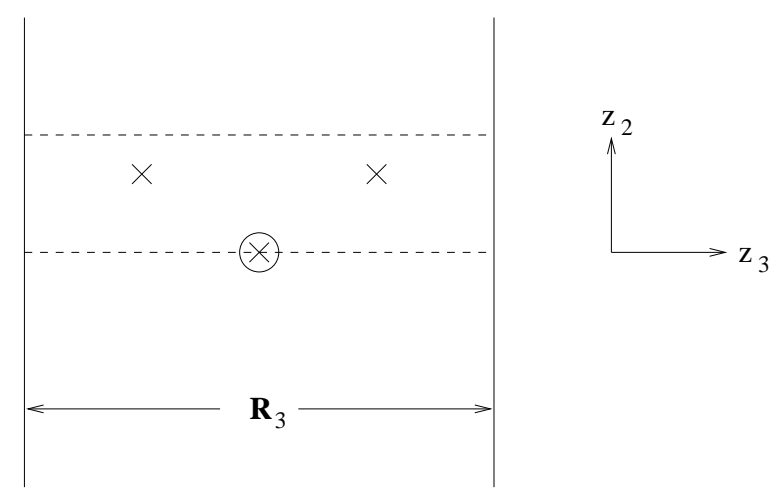

FIG. 1. A schematic diagram of various types of twisted fields. The complex coordinate $z_{1}$ is suppressed in this diagram. The $\mathbb{Z}_{3}$ and $\mathbb{Z}_{6}$ twisted fields are localized at fixed points indicated by crosses and a circle respectively. The $\mathbb{Z}_{2}$ twisted field are localized at fixed loci indicated by dotted lines. The two solid lines which are separated by $R_{3}$ (the size of the compactified dimensions in the $z_{3}$ direction) are identified. Our brane world is the dotted line with $z_{1}=z_{2}=0$ and so the $\mathbb{Z}_{6}$ fixed point is localized on our brane.

In more realistic string models, there can be more than one type of twisted fields. In particular, there can be some twisted fields which are located at fixed loci rather than fixed points in the internal space. For example, in the $\mathbb{Z}_{6}$ orbifold 18 generated by the above $\mathbb{Z}_{3}$ twist and an additional $\mathbb{Z}_{2}$ twist where the $\mathbb{Z}_{2}$ generator $R$ acts on the coordinates as follows: $R z_{1}=-z_{1}, R z_{2}=-z_{2}$, and $R z_{3}=z_{3}$. The $\mathbb{Z}_{2}$ twisted sector states are located at 16 fixed loci in the internal space with $z_{1}$ and $z_{2}$ given by $\left(z_{1}, z_{2}\right)=\frac{1}{2}\left(\left(a_{1}+i b_{1}\right) R_{1},\left(a_{2}+i b_{2}\right) R_{2}\right)$ where $a_{i}, b_{i}=0,1$. While the $\mathbb{Z}_{2}$ twisted fields have no momentum or winding in the complex dimensions $z_{1}$ and $z_{2}$, they are free to propagate in the complex dimension not twisted by the $\mathbb{Z}_{2}$ generator, i.e., $z_{3}$. Therefore, the $\mathbb{Z}_{2}$ twisted sector states live in six dimensions. Even for the $\mathbb{Z}_{2}$ twisted states that sit on the branes (the ones with $a_{2}=b_{2}=0$ ), the corresponding couplings are suppressed by the volume of the compactified dimensions in which the $\mathbb{Z}_{2}$ twisted sector states are free to propagate. In other words, $\kappa \sim g_{s} /\left(V_{3} M_{s}^{2}\right)$ where $V_{3}$ is the volume of the compactified dimensions not twisted by $\mathbb{Z}_{2}$. In addition to the $\mathbb{Z}_{3}$ and $\mathbb{Z}_{2}$ twisted fields described above, there are also $\mathbb{Z}_{6}$ twisted sector states. A schematic diagram of various types of twisted fields is given in Figure 1.

The gauge group of this model is $\left[S U(6) \otimes S U(6) \otimes S U(4) \otimes U(1)^{3}\right]^{2}$. Although this contains the SM, the residual gauge symmetry is too large for the model to be phenomenologically interesting. The rank of the gauge group can be reduced by turning on the untwisted NS-NS sector B-field background [19]. In particular, a three-family Pati-Salam like model was constructed in this framework [4]. Some other four-dimensional $\mathcal{N}=1$ Type I string models were presented in Ref. [20]. As noted above, the twisted fields are massless before SUSY is broken, but would pick up masses after SUSY breaking and dilaton stabilization.

The coupling of the twisted R-R fields $a_{k}$ to a $U(1)$ gauge field is proportional to $\operatorname{Tr}\left(\gamma_{k} \lambda_{i}\right) \partial_{\mu} a_{k} A_{i}^{\mu}$, where $\lambda_{i}$ is the Chan-Paton wavefunction of the abelian gauge field $A_{i}^{\mu}$, and $\gamma_{k}$ defines the action of the orbifold on the D-branes. The coupling of $a_{k}$ with a pair of gauge fields (abelian or non-abelian) is $\propto \operatorname{Tr}\left(\gamma_{k}^{-1} \lambda_{G}^{2}\right) a_{k} F \tilde{F}$ where $\lambda_{G}$ is the Chan-Paton wavefunction of the gauge fields with gauge group $G$. Here, $F(\tilde{F})$ is the field strength 
(dual field strength) for the abelian or non-abelian gauge fields. If $G$ is strongly coupled, the twisted R-R fields $a_{k}$ can play the role of axions. In contrast to perturbative heterotic string theory, a generic Type I string model can have more than one anomalous $U(1)$ [23]. Their triangle anomalies are cancelled by the generalized Green-Schwarz mechanism [21] which involves the exchange of the R-R axions. It is well known that the coupling which mixes a R-R axion with a $U(1)$ gauge field is part of the $U(1)$ gauge-invariant combination $\frac{1}{2} M_{s}^{2}\left(\partial_{\mu} a_{i}-g_{i} e_{i} A_{i}^{\mu}\right)^{2}$ where $A_{i}^{\mu}, i=1,2, \ldots N$ are the anomalous $U(1)$ 's, $g_{i}$ are the gauge couplings and $e_{i}$ are the corresponding charges. The $N U(1)$ gauge bosons become massive, eating up $N$ of the twisted R-R fields (more precisely, $N$ linear combinations of the twisted R-R fields $a_{k}$ described above).

Of the remaining twisted RR fields, it is likely that all except one are relatively heavy. Although we do not expect continuous global symmetry in a string model, it is plausible that some approximate global symmetries are present in the low energy effective field theory, i.e., such symmetries are broken only by high-dimensional operators in the effective theory. If an approximate Peccei-Quinn symmetry is present, effectively, we have an axion field. In this situation, strong interaction chiral symmetry dictates its properties, that is, its mass and coupling follow from its mixing with the neutral pion, with the mixing given by $f_{\pi} / M_{s}$. For $M_{s} \sim 10 \mathrm{TeV}$, this is clearly ruled out by experiments [24]. However, typically, this axion field will also couple to other non-abelian gauge fields in the hidden sector, and their gauge couplings are expected to be strong at relatively high scales (compared to the QCD scale). In this situation, the axion will pick up a relatively large mass and so can avoid detection [25].

Let us now focus on the couplings of the twisted states with the SM fields. The bosonic states from the twisted sectors have spin-0, and so can couple to a pair of gauge fields. In particular, the above mentioned couplings of the $\mathrm{R}-\mathrm{R}$ axions with a pair of gauge fields belong to this type. Similarly, the spin-0 fields from the NS-NS sectors also couple to the gauge fields, in the form

$$
\operatorname{Tr}\left(\gamma_{k}^{-1} \lambda_{G}^{2}\right) \phi_{k} F^{2} .
$$

The spin-0 fields from the twisted sectors can also couple to a pair of fermions $\psi_{L}$ and $\chi_{R}$ with opposite chirality. Since the twisted fields (denoted collectively as $T$ ) are gauge singlets, the three-point coupling to the Standard Model fermions $T \bar{\psi}_{L} \chi_{R}$ is absent, since it is not a Standard Model singlet. However, the lowest order non-renormalizable couplings, such as

$$
\mathcal{C}_{Q d} \frac{g_{s}^{3 / 2}}{M_{s}} \bar{Q}_{L} d_{R} H_{d} T, \quad \mathcal{C}_{Q u} \frac{g_{s}^{3 / 2}}{M_{s}} \bar{Q}_{L} u_{R} H_{u} T, \quad \mathcal{C}_{L \ell} \frac{g_{s}^{3 / 2}}{M_{s}} \bar{L}_{L} \ell_{R} H_{d} T
$$

are presumably allowed 凹, are presumably allowed, where, in standard notation, $Q=\left(\begin{array}{l}u \\ d\end{array}\right)$, $L=\left(\begin{array}{c}\nu_{\ell} \\ \ell\end{array}\right), H_{d, u}$ are the $Y=1,-1$ Higgs in the MSSM, and we suppress generation labels

\footnotetext{
${ }^{1}$ The powers of $g_{s}$ can be seen as follows. The coupling between three open string states is proportional to $g_{s}^{1 / 2}$ whereas the coupling of one closed and two open string states is proportional to $g_{s}$. The above 4-point terms can be factorized into a product of these two types of three-point couplings.
} 
(the $\mathcal{C}$ 's are dimensionless coupling matrices in generation space). There are two types of cubic couplings that result from these quartic couplings. First, nonperturbative effects can generate a nonzero potential for the $T$ fields and hence they can acquire non-zero vevs. These produce the cubic couplings

$$
\mathcal{C}_{Q d} \frac{g_{s}^{3 / 2}}{M_{s}} \bar{Q}_{L} d_{R} H_{d}\langle T\rangle, \quad \mathcal{C}_{Q u} \frac{g_{s}^{3 / 2}}{M_{s}} \bar{Q}_{L} u_{R} H_{u}\langle T\rangle, \quad \mathcal{C}_{L} \frac{g_{s}^{3 / 2}}{M_{s}} \bar{L}_{L} \ell_{R} H_{d}\langle T\rangle
$$

These would combine with the original dimension-4 Yukawa operators, and the generational/flavor structure of the matrices $\mathcal{C}$ may help to yield the observed hierarchical fermion masses and quark mixing. Second, when $H_{u}$ and $H_{d}$ pick up vevs, the quartic operators in (3) will yield the cubic operators

$$
\mathcal{C}_{Q d} \frac{g_{s}^{3 / 2}}{M_{s}} \bar{Q}_{L} d_{R}\left\langle H_{d}\right\rangle T, \quad \mathcal{C}_{Q u} \frac{g_{s}^{3 / 2}}{M_{s}} \bar{Q}_{L} u_{R}\left\langle H_{u}\right\rangle T, \quad \mathcal{C}_{L \ell} \frac{g_{s}^{3 / 2}}{M_{s}} \bar{L}_{L} \ell_{R}\left\langle H_{d}\right\rangle T
$$

For simplicity, let us neglect mixing effects. If the $\mathcal{C}$ have the structure of the quark and lepton mass hierarchies, $\sim m_{f} / M_{e w}<<1$ for all fermions $f$ other than the top quark, then the induced 3-point couplings in (5) are presumably rather small. Note that in the PatiSalam case in Ref [A], the right-handed fermion components $f_{R}$ are $S U(2)_{R}$ doublets, which generically gives rise to a further suppression factor $\propto M_{R} / M_{s}$, where $M_{R}$ denotes the mass of the $W_{R}$ vector boson.

Thus, some linear combinations of the twisted RR scalars are eaten by the anomalous $U(1)$ gauge bosons. The remaining ones $a_{i}$ together with the twisted NS-NS scalars $\phi_{i}$ can appear in the low energy effective action. Some of the lowest order terms in the effective Lagrangian involving the couplings of these twisted modes or the anomalous $U(1)$ gauge fields with the SM fields are:

$$
\begin{aligned}
\mathcal{L} & =\frac{g_{s}}{M_{s}}\left(\sum_{i, j} \mathcal{C}_{i j}^{(1)} \phi_{i} F_{j}^{2}+\sum_{i, j} \mathcal{C}_{i j}^{(2)} a_{i} F_{j} \tilde{F}_{j}\right)+\frac{g_{s}^{3 / 2}}{M_{s}}\left(\bar{f}_{L} f_{R} H \sum_{i, f} \mathcal{C}_{i}^{(3)} a_{i}+\bar{f}_{L} f_{R} H \sum_{i, f} \mathcal{C}_{i}^{(4)} \phi_{i}\right) \\
& +\left(\sum_{i, f} g_{i} e_{L i} \bar{f}_{L} \gamma_{\mu} f_{L} A_{i}^{\mu}+\sum_{i, f} g_{i} e_{R i} \bar{f}_{R} \gamma_{\mu} f_{R} A_{i}^{\mu}\right)+\ldots
\end{aligned}
$$

where $\bar{f}_{L} f_{R} H=\bar{Q}_{L} d_{R} H_{d}, \bar{Q}_{L} u_{R} H_{u}, \bar{L}_{L} \ell_{R} H_{d}$, generation indices are suppressed, $\mathcal{C}_{i j}^{(k)}$ and $\mathcal{C}_{i}^{(k)}$ are model-dependent coefficients, $A_{i}^{\mu}$ denote the anomalous $U(1)$ gauge fields, with gauge couplings $g_{i}$, and $e_{i}^{L}$ and $e_{i}^{R}$ are the corresponding charges. $F_{j}^{2}$ and $F_{j} \tilde{F}_{j}$ sum over abelian and nonabelian gauge fields. The nonperturbative effects that generate a nonzero potential for the moduli (as is necessary for dilaton stablization and is related to SUSY breaking) will, in general, produce various quadratic, cubic, and quartic couplings involving these moduli. These are subject to obvious constraints; for example, they must be invariant under the orbifold twists.

In this letter, we discuss Type I strings on orbifolds, and more generally, when the orbifold singularities are smoothed out ("blown-up") to a Calabi-Yau manifold as the twisted NS-NS scalars $\phi_{k}$ acquire non-zero vevs. The coupling of the twisted modes with the brane fields is $\kappa \sim g_{s} f\left(\left\langle\phi_{k}\right\rangle\right)$ where $f\left(\left\langle\phi_{k}\right\rangle\right)$ is a function of $\left\langle\phi_{k}\right\rangle$. Even in the generic case where $\left\langle\phi_{k}\right\rangle$ is 
comparable with the string scale, it is likely that the suppression factor for the bulk fields, i.e., $\left(V_{p-3} V_{9-p} M_{s}^{6}\right)^{-1}$ is still much smaller than $f\left(\left\langle\phi_{k}\right\rangle\right)$ and so there are closed string fields which couple to the brane fields more strongly than the bulk fields. Moreover, we expect that the features we discuss here would still apply for other realizations of the brane world (which may be viewed as dual to the Type I description), such as non-perturbative heterotic string/M theory models with solitonic fivebranes [27] as well as F-theory [28] models.

\section{EXPERIMENTAL SIGNATURES AND CONSTRAINTS}

There are several experimental implications of eq. (6). In general, there could be couplings such as $g_{s} \mu_{i j k} T_{i} T_{j} T_{k}$, where the coefficient $\mu_{i j k}$ would presumably be of order the electroweak scale. These would allow a subset of the $\xi=\phi_{k}, a_{k}$ to decay rapidly, with widths $\Gamma \sim g_{s}^{2} \mu^{2} / m_{\xi}$. We concentrate here on the light $\xi$ fields for which these decay channels are kinematically forbidden, as well as the subset of the heavier $\xi$ fields whose analogous decays do not occur because the corresponding cubic or quartic operators are absent (as a consequence, for example, of noninvariance under the orbifold twists).

In the following, we refer collectively to the various mass eigenstates of $\phi$ and $a$ and suppress their multiplicity; similarly, we use $\mathcal{C}$ to refer to the appropriate linear combinations of the original $\mathcal{C}_{i j}^{(1)}$ and $\mathcal{C}_{i j}^{(2)}$ for these mass eigenstates. The masses $m_{\xi}, \xi=\phi, a$, are expected to be of roughly of order the SUSY breaking scale, which is comparable to the electroweak symmetry breaking scale. The couplings in (6) give rise to the decays $\xi \rightarrow g g$ as well as $\xi \rightarrow \gamma \gamma$, and (if kinematically allowed) $\xi \rightarrow Z Z, W^{+} W^{-}$. From the lowest-order graphs, in terms of

$$
\Gamma_{0}=\frac{g_{s}^{2} \mathcal{C}^{2}}{64 \pi}\left(\frac{m_{\xi}}{M_{s}}\right)^{2} m_{\xi}
$$

we find $\Gamma(\xi \rightarrow g g)=\left(N_{c}^{2}-1\right) \Gamma_{0}$ where $N_{c}=3, \Gamma(\xi \rightarrow \gamma \gamma)=\Gamma_{0}, \Gamma(\xi \rightarrow Z Z)=\Gamma_{0}(1-$ $\left.4 m_{Z}^{2} / m_{\xi}^{2}\right)^{1 / 2}$, and $\Gamma\left(\xi \rightarrow W^{+} W^{-}\right)=2 \Gamma_{0}\left(1-4 m_{W}^{2} / m_{\xi}^{2}\right)^{1 / 2}$, with $\xi=\phi, a$. Since one expects $g_{s} \sim O(1)$, these lowest-order calculations are only rough estimates. Summing the partial widths and taking $g_{s} \sim O(1), \mathcal{C} \sim O(1)$, and $m_{W, Z}^{2} / m_{\xi}^{2}<<1$, we get

$$
\Gamma_{\xi} \sim 0.1\left(m_{\xi} / M_{s}\right)^{2} m_{\xi}
$$

For $m_{\xi}=1 \mathrm{TeV}, M_{s}=10 \mathrm{TeV}$, this gives $\Gamma_{\xi} \sim 10^{-3} m_{\xi} \sim 1 \mathrm{GeV}$. The dominant decays of the $\phi, a$, namely $(\phi, a) \rightarrow g g$, should not involve large missing energy and should in principle allow one to reconstruct the mass.

The couplings $\phi F^{2}$ and $a F \tilde{F}$ with the gluon field in (6) contribute to single jet inclusive, dijet, and multijet production in $\bar{p} p$ (and, equally, in $p p$ ) collisions. The $\xi$ can be produced in the process $g g \rightarrow g \xi$ involving the usual triple Yang-Mills vertex multiplied by $g_{s} \mathcal{C} / M_{s}$. The resultant decay of the $\xi$ yields 3 -jet events. The rate, relative to regular QCD 3-jet events is roughly $\left(g_{s} \mathcal{C} / \alpha_{3}\right)^{2}\left(\hat{s} / M_{s}\right)^{2}\left(1-m_{\xi}^{2} / \hat{s}\right)^{1 / 2}$ (where $\hat{s}$ is the $g g$ center of mass energy squared) which could be $O\left(10^{-2}\right)$ for $\sqrt{s}=1.8 \mathrm{TeV}$ and our illustrative values of $g_{s}, M_{s}, m_{\xi}$.

In $g g \rightarrow g g$ scattering, there are new contributions from graphs involving the coupling of $g g$ to $\xi=\phi, a$ in the $s, t$ and $u$ channels. From the tree-level graphs, we compute the new contribution to $g g \rightarrow g g$ to be $d \sigma / d \hat{t}=|\mathcal{M}|^{2} /\left(16 \pi \hat{s}^{2}\right)$ where 


$$
\begin{aligned}
& \bar{\sum}|\mathcal{M}|^{2}=\frac{\left(N_{c}^{2}-1\right)}{16}\left(\frac{g_{s} \mathcal{C}}{M_{s}}\right)^{4} \sum_{m=m_{\phi}, m_{a}}\left[\frac{\hat{s}^{4}}{\left(\hat{s}-m^{2}\right)^{2}}+\frac{\hat{t}^{4}}{\left(\hat{t}-m^{2}\right)^{2}}+\frac{\hat{u}^{4}}{\left(\hat{u}-m^{2}\right)^{2}}+\right. \\
& \left.\frac{\hat{s}^{4}+\hat{t}^{4}+\hat{u}^{4}-2 \hat{u}^{2}\left(\hat{s}^{2}+\hat{t}^{2}\right)}{2\left(\hat{s}-m^{2}\right)\left(\hat{t}-m^{2}\right)}+\frac{\hat{s}^{4}+\hat{t}^{4}+\hat{u}^{4}-2 \hat{s}^{2}\left(\hat{t}^{2}+\hat{u}^{2}\right)}{2\left(\hat{t}-m^{2}\right)\left(\hat{u}-m^{2}\right)}+\frac{\hat{s}^{4}+\hat{t}^{4}+\hat{u}^{4}-2 \hat{t}^{2}\left(\hat{s}^{2}+\hat{u}^{2}\right)}{2\left(\hat{s}-m^{2}\right)\left(\hat{u}-m^{2}\right)}\right]
\end{aligned}
$$

where integration over final state phase space involves a $(1 / 2)$ factor for identical particles. (As indicated, the $\phi F^{2}$ and $a F \tilde{F}$ contributions are of the same form, with $m_{\phi} \rightarrow m_{a}$.) Since $g_{s} \sim O(1)$, higher order corrections can be substantial; however, (9) gives a rough estimate. For $\hat{s} \simeq m^{2}$, the $s$-channel term has a strong resonant enhancement (since the $s$-channel propagator is actually $\propto 1 /(m \Gamma)$ at $\left.\hat{s}=m^{2}\right)$, and, in particular, the $M_{s}^{-4}$ factor from the coupling is cancelled by the $M_{s}^{4}$ factor in $\Gamma_{\xi}^{-2}$ arising from the square of the $s$ channel propagator. Inserting (8), we find that $\bar{\sum}|\mathcal{M}|^{2} \simeq 50\left(g_{s} \mathcal{C}\right)^{4}$. This is comparable to the regular QCD contribution, which, e.g., for $g g \mathrm{CM}$ angle $\hat{\theta}=\pi / 2$, gives $\bar{\sum}|\mathcal{M}|^{2} \simeq 30 g_{3}^{4}$, where $g_{3}$ is the $\mathrm{SU}(3)$ coupling. The most striking effect is that there would be peaks in the dijet invariant mass at $M_{J J}=m_{\phi, a}$. We recall that there are, in general, several $\phi_{k}$ and $a_{k}$, so there could be several such resonances. Let us compare the size of this effect with the conventional scenario in which $M_{s} \sim M_{P}$. In that case, the moduli fields $\phi, a$ could also be of order the SUSY breaking scale, and, given that $M_{s}$ cancels out at the resonance for $\hat{s}=m_{\xi}^{2}$, the existence of this resonance, per se, would not constitute evidence of low-string scale, as opposed to conventional $M_{s} \sim M_{P}$ models; however, the contribution of (9) to $d^{2} \sigma / d M_{J J} d \cos \hat{\theta}$ involves the convolution over the momentum fractions $x_{1}, x_{2}$ of the gluons, and the off-resonant contributions to this convolution would be very different for low- and high-string scale theories because of the quite different $M_{s}$ scales.

Current data on 2-jet and inclusive jet production in $\bar{p} p$ collisions from D0 is in excellent agreement with QCD predictions [26]. This is also true of the CDF data in the region where the $g g \rightarrow g g$ subprocess makes its main contribution, for $E_{T} \lesssim 300 \mathrm{GeV}$ [29] (the latter resulting from the rapid rise of the gluon distribution functions $g(x)$ for small $x$ and the fact that $\left.E_{T}^{2}=(\hat{s} / 4) \sin ^{2} \hat{\theta}<x_{1} x_{2} s / 4\right)$. Although the new contributions from $\phi, a$ exchange can only roughly be estimated, in view of the expected large $g_{s} \sim O(1)$ and the modeldependent factor $\mathcal{C}$, we infer that if $\mathcal{C} \sim 1$, then a safe bound in order to avoid conflict with this data is $m_{\phi, a} \gtrsim O(1) \mathrm{TeV}$. Furthermore, if $m_{\phi, a}$ are sufficiently large so that there is no significant resonant contribution, the data would still constrain $M_{s} \gtrsim O(1) \mathrm{TeV}$, given that the corresponding couplings $g_{s} \mathcal{C} \sim O(1)$. In models with a low string scale and large compactification radii, there will also be a new contributions to $\bar{p} p$ scattering from processes involving the exchange or emission of gravitational KK modes; however, in a global data analysis, one could still distinguish between the effects of these KK modes and of the $\phi, a$.

Besides the contribution of the $\phi_{k} F_{j}^{2}$ term to gauge couplings, via $\left\langle\phi_{k}\right\rangle$, these terms also contribute to various loop effects which could be significant. Another important implication concerns neutral vector bosons. In contrast to perturbative heterotic string theory, there can be several anomalous $U(1)$ gauge symmetries in Type I string models. Moreover, in perturbative heterotic string theory where $M_{s} \sim M_{P}$, the associated anomalous $U(1)$ vector bosons are too heavy to be detected. However, the anomalous $U(1)$ vector bosons in the present scenario can be relatively light $(\lesssim$ few $\mathrm{TeV})$, and are possible candidates for $Z^{\prime}$ bosons. These vector bosons gain masses by "eating" some of the above axions. The resultant $Z^{\prime}$ 's couple to the brane fields with strength comparable with SM interactions. 
Current lower bounds on such $Z^{\prime}$ bosons are of order $800 \mathrm{GeV}$, depending on their couplings [30].

In addition to the scalar component fields $\phi, a$, the twisted moduli chiral superfield $\mathcal{T}$ also has modulino component fields $\tilde{\phi}, \tilde{a}$. We denote these collectively as $\tilde{T}$. Clearly these would play the role of a electroweak singlet ("sterile") neutrinos. Once a nonflat superpotential is generated (nonperturbatively) for the twisted moduli, there would be terms of the form $\mathcal{T} \mathcal{T}$ yielding the bilinears $\tilde{T}^{T} C \tilde{T}$, with mass coefficients presumably of order the SUSY breaking scale and hence comparable to the electroweak scale. If allowed by the matter parities resulting from the underlying string theory, there could also be cubic $\mathcal{L} \mathcal{T H}$ terms in the superpotential, which would give rise to Dirac neutrino mass terms of the form $\bar{\nu}_{L} \tilde{T}_{R}$. Note that the $\tilde{T}$ fields couple to brane fields, in particular, neutrinos, without the volume suppression factors affecting the couplings of the bulk fields to brane fields. We shall discuss this further elsewhere. This contrasts with the situation for candidates for sterile neutrinos coming from the bulk [8].

The role of a generation dependent anomalous $U(1)$ gauge symmetry in the observed hierarchical fermion mass structure has been studied in the context of perturbative heterotic string theory [31]. An important difference here is that there can be several anomalous $U(1)$ 's (for example, the $Z_{6}$ model discussed earlier has 4 anomalous $U(1)$ 's), and different families have different $U(1)$ charge assignments, as in the case in Ref 团. Hence the possibilities in the brane world are more intricate.

The numerous testable implications of models with low string scale are clearly of great interest and deserve further theoretical and experimental investigation.

\section{ACKNOWLEDGMENTS}

We thank Philip Argyres, Zurab Kakushadze and Piljin Yi for discussions. The research of G.S. and R.S. is partially supported by the NSF grant PHY-97-22101. The research of S.-H.H.T. is partially supported by the NSF. 


\section{REFERENCES}

[1] N. Arkani-Hamed, S. Dimopoulos and G. Dvali, Phys. Lett. B429 (1998) 263.

[2] J. Lykken, Phys. Rev. D54 (1996) 3693.

[3] I. Antoniadis, N. Arkani-Hamed, S. Dimopoulos and G. Dvali, Phys. Lett. B436 (1998) 257.

[4] G. Shiu and S.-H.H. Tye, Phys. Rev. D58 (1998) 106007.

[5] Z. Kakushadze and S.-H.H. Tye, hep-th/9809147.

[6] N. Arkani-Hamed, S. Dimopoulos and G. Dvali, hep-ph/9807344.

[7] K.R. Dienes, E. Dudas and T. Gherghetta, Phys. Lett. B436 (1998) 55; hepph/9806292; hep-ph/9807522. See also, Z. Kakushadze, hep-th/9811193; C.D. Carone, hep-ph/9902407; A. Delgado and M. Quiros, hep-ph/9903400; P.H. Frampton and A. Rasin, hep-ph/9903479.

[8] N. Arkani-Hamed, S. Dimopoulos, G. Dvali and J. March-Russell, hep-ph/9811448; K.R. Dienes, E. Dudas and T. Gherghetta, hep-ph/9811428; G. Dvali and A. Yu. Smirnov, hep-ph/9904211.

[9] N. Arkani-Hamed and S. Dimopoulos, hep-ph/9811353; Z. Berezhiani and G. Dvali, hep-ph/9811378.

[10] P.C. Argyres, S. Dimopoulos and J. March-Russell, hep-th/9808138; K.R. Dienes, E. Dudas, T. Gherghetta and A. Riotto, hep-ph/9809406; N. Arkani-Hamed, S. Dimopoulos and J. March-Russell, hep-th/9809124; N. Kaloper and A. Linde, hep-th/9811141; G. Dvali and S.-H.H. Tye, hep-ph/9812483; N. Arkani-Hamed, S. Dimopoulos, N. Kaloper, J. March-Russell, hep-ph/9903224,hep-ph/9903239; G. Dvali and G. Gabadadze, hepph/9904221, and references therein.

[11] A. Pomarol and M. Quirós, Phys. Lett. B438 (1998) 255; C.P. Bachas, hep-ph/9807415; D. Ghilencea and G.G. Ross, hep-ph/9809217; I. Antoniadis, S. Dimopoulos, A. Pomarol and M. Quirós, hep-ph/9810410; T. Kobayashi, J. Kubo, M. Mondragon and G. Zoupanos, hep-ph/9812221; G. Domokos and S. Kovesi-Domokos, Phys. Rev. Lett. 82 (1999) 1366; A.E. Faraggi and M. Pospelov, hep-ph/9901299; T. Banks, M. Dine and A.E. Nelson, hep-th/9903019.

[12] Z. Kakushadze, hep-th/9812163; hep-th/9902080.

[13] G.F. Giudice, R. Rattazzi and J.D. Wells, Nucl. Phys. B544 (1999) 3; S. Nussinov and R. Shrock, Phys. Rev. D59, (1999) 105002; E.A. Mirabelli, M. Perelstein and M.E. Peskin, Phys. Rev. Lett. 82, (1999) 2236; T. Han, J.D. Lykken and R.-J. Zhang, hepph/9811350; J.L. Hewett, hep-ph/9811356; P. Mathews, S. Raychaudhuri and K. Sridhar, hep-ph/9811501; hep-ph/9812486; T.G. Rizzo, hep-ph/9901209; hep-ph/9902273; hep-ph/9903475 K. Agashe and N.G. Deshpande, hep-ph/9902263; K. Cheung and W.Y. Keung, hep-ph/9903294; D. Atwood, S. Bar-Shalom and A. Soni, hep-ph/9903538; C. Balazs, H.-J. He, W.W. Repko, C.-P. Yuan and D.A. Dicus, hep-ph/9904220.

[14] M.L. Graesser, hep-ph/9902310; P. Nath and M. Yamaguchi, hep-ph/9902323, hepph/9903298.

[15] S. Cullen and M. Perelstein, hep-ph/9903422.

[16] I. Antoniadis, Phys. Lett. B246 (1990) 377; I. Antoniadis, C. Muñoz and M. Quirós, Nucl. Phys. B397 (1993) 515; I. Antoniadis, K. Benakli and M. Quirós, Phys. Lett. B331 (1994) 313; I. Antoniadis, S. Dimopoulos and G. Dvali, Nucl. Phys. B516 (1998) 70. 
[17] C. Angelantonj, M. Bianchi, G. Pradisi, A. Sagnotti and Ya.S. Stanev, Phys. Lett. B385 (1996) 96.

[18] Z. Kakushadze and G. Shiu, Nucl. Phys. B520 (1998) 75.

[19] M. Bianchi, G. Pradisi and A. Sagnotti, Nucl.Phys. B376 (1992) 365; M. Bianchi, Nucl. Phys. B528 (1998) 73; E. Witten, JHEP 9802 (1998) 006; Z. Kakushadze, G. Shiu and S.-H.H. Tye, Phys. Rev. D58 (1998) 086001.

[20] Z. Kakushadze, Nucl. Phys. B535 (1998) 311; Z. Kakushadze and S.-H.H. Tye, Phys. Rev. D58 (1998) 126001.

[21] M. Green and J. Schwarz, Phys. Lett. B149 (1984) 117.

[22] M. Dine, N. Seiberg and E. Witten, Nucl. Phys. B289 585 (1987); M.R. Douglas and G. Moore, hep-th/9603167.

[23] A. Sagnotti, Phys. Lett. B294 (1992) 196; L.E. Ibáñez, R. Rabadán and A.M. Uranga, Nucl. Phys. B542 (1999) 112; L.E. Ibáñez, C. Muñoz and S. Rigolin, hep-ph/9812397; M. Cvetic, L. Everett, P. Langacker, J. Wang, hep-th/9903051; Z. Lalak, S. Lavignac, H.P. Nilles, hep-th/9903160.

[24] See e.g., J.E. Kim, Phys. Rep. 1501 (1987).

[25] S.-H.H. Tye, Phys. Rev Lett. 471035 (1981)

[26] B. Abbott et al., (D0 Collaboration), Phys. Rev. Lett. 82, 2451, 2457 (1999) (hepex/9807014, hep-ex/9807018).

[27] See, e.g., R. Donagi, A. Lukas, B.A. Ovrut and D. Waldram, hep-th/9811168, hepth/9901009.

[28] C. Vafa, Nucl. Phys. B469 (1996) 403; D.R. Morrison and C. Vafa, Nucl. Phys. B473 (1996) 74; Nucl Phys. B476 (1996) 437.

[29] G. Blazey and B. Flaugher, hep-ex/9903058 and references therein.

[30] Particle Data Group, Review of Particle Physics, Eur. J. Phys. C3 (1998).

[31] L.E. Ibáñez and G. G. Ross, Phys. Lett. B332, (1994) 100; V. Jain and R. Shrock, Phys. Lett. B352 (1995) 83; P. Binetruy and P. Ramond, Phys. Lett. B350, (1995) 49. 